\title{
Comparison of the Effectiveness of Schema Therapy and Dialectical Behavior Therapy on Temperament and Character Dimensions and Cognitive Emotion
}

\section{Regulation Strategies in Patients with HIV}

\author{
Vahid Hemmati Sabet ${ }^{1}$, Saeedeh Alsadat Hoseini ${ }^{2 *}$, Karim Afsharinia ${ }^{3}$, Mokhtar Arefi ${ }^{4}$ \\ 1. Department of Psychology, Kermanshah Branch, Islamic Azad University, Kermanshah, Iran. \\ 2. Department of Psychology, Kermanshah Branch, Islamic Azad University, Kermanshah, Iran. \\ 3. Department of Psychology, Kermanshah Branch, Islamic Azad University, Kermanshah, Iran. \\ 4. Department of Psychology, Kermanshah Branch, Islamic Azad University, Kermanshah, Iran. \\ * Corresponding author's Email: $\underline{\text { saeedeh_hosseini@iauksh.ac.ir }}$
}

\begin{abstract}
There are many social concerns towards HIV disease and people feel irrational fears of infection and modes of virus transmission. These factors might affect the temperament and character dimensions, as well as cognitive emotion regulation strategies. Accordingly, psychological therapies can be used as beneficial tools to identify the disease and to mitigate the related concerns. This study aimed at comparing the effectiveness of Schema Therapy (ST) and Dialectical Behavior Therapy (DBT) on temperament and character (TC) dimensions and cognitive emotion regulation (CER) strategies in patients with HIV. In terms of methodology, this was an experimental study based on the pretest-posttest and control group. Also, this was applied research in terms of objective. The statistical population comprised all patients with HIV who were under treatment in behavioral disorders clinics of Health Centers in Hamedan, Iran, during 2019-2020. Of them, 45 subjects were chosen by using simple random sampling then were assigned to three groups $(n=15)$, including two intervention groups and one control group. To assess the considered variables, the Temperament and Character Inventory (TCI) designed by Cloninger (1994), and the Cognitive Emotion Regulation Questionnaire (CERQ) (2001) were used. According to ANCOVA results, ST and DBT affected temperament and character dimensions and there was a significant difference between the two groups. Therefore, although both therapies had effectiveness in surveyed variables, ST had higher effectiveness rather than DBT.
\end{abstract}

Keywords: Schema Therapy, Dialectical Behavior Therapy, Temperament and Character, Cognitive Emotion Regulation Strategies, HIV

\section{Introduction}

As a severe disease, HIV is a critical matter due to its many complications, such as mortality, high financial costs caused by treatment and care, social and psychological problems like depression, and many other issues (Cane, 2018). There has been an increase in the number of patients with HIV whose risky behaviors play a vital role in this trend (King \& Winchester, 2018). Moreover, due to different personality traits and subsequently, the application of various coping strategies for this disease, the temperament, and character of individuals take a major part in this case (Ashraf \& Sitwat, 2016). Personality growth is based on the interaction between temperament and character dimensions. Temperament is the biological and heritable dimension of personality, and character implies its environmental dimension (Svrakic \& Cloninger, 2012). Many studies have proved the relationship of 
HIV with personality and its various dimensions. For instance, a study indicated that patients with HIV may suffer from high neurosis (Schadé, Van Grootheest \& Smit, 2013). Besides, cognitive emotion regulation can be named as another factor playing role in adaptation with experiences (Baradaran, 2017). Cognitive emotion regulation is composed of cognitions and cognitive procedures assisting individuals in regulating their feelings and emotions (Rajabi, Nazarpour \& Tabnak, 2017). Emotion regulation describes how individuals experience, modify and organize emotions. Emotion regulation also reveals the effect of this organization on behavior (Sahraian, Peyvastegar, Khosravi \& Habibi, 2019). Emotion regulation strategies and procedures have a considerable effect on health and disease (Gross, 2015). Further, the severity of emotional problems in patients with HIV can be determined concerning their difficulty in emotion regulation (Brandt, Zvolensky, Woods, Gonzalez, Safren \& O'Cleirigh, 2017). Results of many studies have emphasized the relationship between emotion regulation and HIV. For example, findings of a study indicated high emotional stress among women with HIV (FarmaniShahreza, Ghaedniay-jahromi, Mohammad-Taghi Nasab, Niknezhad \& et al., 2017). Since cognitive strategies are substantial emotion regulation strategies and failure in emotion regulation is treated as emotion dysregulation meaning maladaptive methods used by individuals when facing emotional conditions (Sahraian, Peyvastegar, Khosravi \& Habibi, 2019), psychological interventions, such as schema therapy can be effective. According to Schema Therapy Approach developed by Young, early maladaptive schemas are self-defeating cognitive emotion schema that is developed at the beginning stage of mental growth. These maladaptive schemas may occur throughout one's lifetime (Young, Klosko \& Weishaar, 2003). These schemas cause inefficient perceptions and unbalanced behaviors and emotions (Khodabandelow, Najafi \& Rahimian Boogar, 2018). It was concluded in a study that schema therapy contributes to the improvement of cognitive emotion regulation strategies in patients with HIV (Farmani-Shahreza, Ghaedniay-jahromi, Mohammad-Taghi Nasab, Niknezhad \& et al., 2017). A study was conducted on female prisoners who suffer from HIV and results showed that schema therapy-based cognitive therapy could reduce psychological, emotional, and depression problems (Jalali, Hasani, Hashemi, Kimiaei \& Babaei, 2019). In another research, findings implied the positive effectiveness of schema therapy in reducing emotion dysregulation (Dadomo, Panzeri, Caponcello, Carmelita \& Grecucci, 2018). Besides schema therapy, dialectical behavior therapy (DBT) can be used as another effective intervention in which pathological symptoms are examined as maladaptive problem-solving (Teimory, Ghafariyan, \& Yazdanpanah, 2019). In this approach, patients learn how to overcome the conflicts between themselves and the environment achieving a practical outcome by integrating these conflicts (Belir, Ansari Shahidi, \& Mohammadi, 2018). In one research, authors found the effective role of dialectical behavior therapy in mental and psychological problems especially issues related to character and personality disorders (Kröger, Harbeck, Armbrust, \& Kliem, 2013). As a new approach to human nature, personality, and behaviors, dialectical behavioral therapy has remained unknown in Iran; hence, it is essential to conduct a study on this case (Hajializadeh, \& Norizadeh, 2018). Accordingly, this study was conducted to find whether ST and DBT have an effect on TC dimensions 
and CER strategies and whether there is a difference between the effect of ST and DBT on TC dimensions and CER strategies in patients with HIV?

This study provides mental health experts with a high concentration on cognition throughout a certain way in which they can work well on TC dimensions and CER strategies. Moreover, such studies can be used as an effective measure by these experts who need to know this disease more and to mitigate relevant concerns owing to social concerns towards HIV and irrational public fear of infection and effect of virus transmission modes on temperament and character dimensions and cognitive emotion regulation strategies of them. Comparative study of the effects of two therapeutic approaches, including ST and DBT, on TC dimensions and CER of patients with HIV, leads to more proper implications as both approaches have a significant impact on personality disorders and inefficient emotions.

\section{Material and Methods}

This was applied research in terms of objective and had an experimental research plan based on pretestposttest along with control group. The statistical population comprised all patients with HIV who were under treatment in behavioral disorders clinics associated with Health Centers in Hamedan, Iran, during 2019-2020. Simple random sampling was employed as a sampling method in which 45 patients were randomly chosen from Shohada Clinic of Hamedan. The selected patients were assigned to three groups $(n=15)$, including two intervention groups and one control group. Inclusion criteria were as follows: age range of 20-60, minimum education level of diploma, being under HIV treatment, and not being treated psychologically.

\section{Tools}

Temperament and Character Inventory (TCI) designed by Cloninger (1994) includes 125 items. This questionnaire proposes a general model, which assesses normal and abnormal personalities. This inventory evaluates biogenetic temperament and acquired character. ICI includes four temperament dimensions (novelty seeking (NS), harm avoidance (HA), reward dependence (RD), and persistence (PS)) and three character dimensions (self-directedness (SD), cooperativeness (Co), and selftranscendence (ST)). Alpha coefficients of persistence and self-transcendence equaled $44 \%$ and $81 \%$, respectively. The average aloha coefficient was reported to $68 \%$, and the overall alpha coefficient was obtained to 74\% (Basharpour, Etarod, \& Eini, 2017).

The Cognitive Emotion Regulation Questionnaire (CERQ) was designed by Garnefski et al. (2001) to assess cognitive emotion regulation strategies. This is a multi-dimensional and self-report 36-item scale that presents two adult and children versions. CER scale evaluates nine cognitive strategies, including self-blame, acceptance, rumination, positive refocusing, refocus on planning, positive reappraisal, rutting into perspective, catastrophizing, and other-blame.

Structural validity and reliability of this scale were measured in Iranian culture by calculating Cronbach's alpha coefficient for each abovementioned subscale. The obtained coefficients varied between 0.64 and 0.82 (SayyahBargard, Olapour, Ardame, Shahidi, \& Yaghoobi Askarabad, 2014). 
Among questionnaires related to dimensions of temperament (novelty seeking, harm avoidance, reward dependence, and persistence) and character (self-directedness, cooperativeness, self-transcendence), as well as cognitive emotion regulation strategies (positive cognitive emotion regulation and negative cognitive emotion regulation) that were pretested, 30 questionnaires were chosen randomly. The reliability of the selected questionnaires was analyzed by measuring Cronbach's alpha.

In this research, reliability values of temperament's components equaled 0.95, 0.96, 0.94, 0.80 for NS, $\mathrm{HA}, \mathrm{RD}$, and PS, respectively; meanwhile, reliability values of character's components equaled 0.96 , 0.97, 0.96 for SD, CO, ST, respectively.

Moreover, the reliability of components of cognitive emotion regulation was measured; positive cognitive emotion regulation (0.84) and negative cognitive emotion regulation (0.92).

Coordinated with the University of Medical Sciences and Health Department of Hamedan, 45 patients were randomly chosen from the considered center (Shohada Clinic) then were assigned to three groups $(n=15)$, including two intervention groups and one control group. Eight schema therapy sessions were held for intervention group 1, and eight DBT-based sessions were held for intervention group 2. Patients attended 90-minute sessions twice a week (every three days). It worth noting that all patients received some verbal and written information about the research and signed the consent letter; so, they participated in the study informed and ensured confidentiality.

Table 1. Summarized content of ST-based training sessions

Session 1: making relationship, primary assessment, introducing members, explaining group rules, signing therapy contract, diagnosing the problem

Session 2: teaching schemas and coping styles, linking current problems to schemas

Session 3: (cognitive strategies), empathic confrontation, the new definition of evidence approving schema

Session 4: evaluating pros and cons of coping responses, opening negotiation between healthy and schema aspects, challenging schemas, teaching how to design educational cards

Session 5: introducing the logic of empirical technique (fighting against schemas at an emotional level), mental imaging, connecting the past mental imaginations to present time, fantastic dialogues

Session 6: presenting the logic of behavioral techniques, expressing the purpose of behavioral techniques, providing methods to list the behavior, ranking the most problematic behaviors, enhancing motivation for behavioral change

Session 7: behavioral techniques, practicing healthy behaviors by imaging and role-playing, overcoming barriers to behavioral change, and creating outstanding changes in life

Session 8: reviewing the previous sessions, summarizing and concluding, appreciating all group members, and ending sessions. 
Table 2. Summarized content of DBT protocol-based training sessions

Session 1: introducing group rules and mindfulness to members, presenting a rational explanation of mindfulness' advantages, explaining beneficial methods of mental focus, determining different kinds of minds for clients, making clients familiar with training skills and their importance, explaining how to focus on fundamental mindfulness skills

Session 2: teaching three skills to improve mindfulness (what has been changed in me and skill of focusing on the pleasant smell mindful eating raisins and gummy candies, expressing skills of what and how)

Session 3: highlighting the effectiveness of interpersonal skills, teaching preferences and requirements and balancing them, developing self-respect and dominance

Session 4: teaching effectiveness of self-respect, discussing environmental factors affecting interpersonal impact, helping individuals to find inspiring sentences for themselves

Session 5: expressing the structure of the emotional model, teaching the presence of mind when there is an emotional mode, teaching to change emotions by reacting the emotion, strategies to reduce negative emotions, strategies to improve positive events

Session 6: teaching mindfulness in present emotional mode, teaching to change emotions by acting against emotional state Session 7: explaining the importance of distress tolerance skills, introducing tolerance skills and survival in critical conditions

Session 8: teaching tolerance strategies, such as distraction methods, the final evaluation of clients

\section{Ethical Considerations}

This paper was approved in the code of IRCT20120215009014N348 by the Iranian Registry of Clinical Trials. Additionally, this study obtained the ethic code of IR.UMSHA.REC.1398.1063 from Hamedan University of Medical Sciences.

\section{Results}

Multivariate MANCOVA was employed for data analysis. The collected data were analyzed through SPSS19. Table 3 reports the mean and standard deviation (SD) of scores given to subscales of temperament, character, and cognitive emotion regulation in three surveyed groups.

Table 3. Mean and SD of variables in two experimental (intervention) and control groups

\begin{tabular}{|l|l|l|l|l|l|}
\hline Statistical indices & \multicolumn{2}{l|}{ Pretest } & \multicolumn{2}{l|}{ Posttest } \\
\hline Variable & Group & Mean & SD & Mean & SD \\
\hline \multirow{3}{*}{ Novelty seeking } & Schema therapy & 7.67 & 2.26 & 17 & 1.26 \\
\cline { 2 - 6 } & Dialectical behavior therapy & 7.40 & 1.59 & 13.47 & 1.06 \\
\cline { 2 - 6 } & Control & 9.27 & 1.33 & 10.47 & 1.39 \\
\hline \multirow{4}{*}{ Harm avoidance } & Schema therapy & 7.20 & 1.82 & 15.13 & 2.53 \\
\cline { 2 - 6 } & Dialectical behavior therapy & 7.53 & 1.41 & 13.13 & 0.915 \\
\cline { 2 - 6 } & Control & 9 & 1 & 11.20 & 1.55 \\
\hline \multirow{4}{*}{ Reward Dependence } & Schema therapy & 5 & 2.17 & 12.60 & 1.72 \\
\cline { 2 - 6 } & Dialectical behavior therapy & 5.20 & 1.26 & 9.60 & 1.50 \\
\cline { 2 - 6 } & Control & 4.27 & 1.28 & 5.33 & 1.23 \\
\hline \multirow{3}{*}{ Persistence } & Schema therapy & 1.40 & 0.51 & 4.27 & 0.59 \\
\cline { 2 - 6 } & Dialectical behavior therapy & 1.60 & 0.51 & 3.07 & 0.70 \\
\hline
\end{tabular}




\begin{tabular}{|l|l|l|l|l|l|}
\hline & Control & 1.27 & 0.46 & 1.47 & 0.743 \\
\hline \multirow{3}{*}{ Self-Directedness } & Schema therapy & 8.20 & 2.37 & 17.47 & 1.25 \\
\cline { 2 - 5 } & Dialectical behavior therapy & 7.80 & 1.78 & 13.93 & 1.03 \\
\cline { 2 - 5 } & Control & 9.67 & 1.63 & 10.07 & 1.58 \\
\hline \multirow{3}{*}{ Seoperativeness } & Schema therapy & 12.60 & 1.06 & 19.40 & 1.72 \\
\cline { 2 - 5 } & Dialectical behavior therapy & 12.13 & 0.915 & 16 & 1.07 \\
\cline { 2 - 5 } & Control & 12.33 & 0.900 & 11 & 1.134 \\
\hline \multirow{3}{*}{ Positive cognitive emotion regulation } & Schema therapy & 5 & 1.77 & 12.53 & 1.88 \\
\cline { 2 - 6 } & Dialectical behavior therapy & 5.27 & 1.28 & 10 & 1.51 \\
\cline { 2 - 6 } & Control & 4.67 & 1.34 & 12.80 & 1.08 \\
\hline \multirow{3}{*}{ Negative cognitive emotion regulation } & Schema therapy & 38.80 & 2.43 & 50.07 & 3.17 \\
\cline { 2 - 6 } & Dialectical behavior therapy & 42.47 & 0.834 & 47.60 & 0.910 \\
\cline { 2 - 6 } & Control & 40.40 & 2.10 & 40.93 & 2.19 \\
\hline & Schema therapy & 54.13 & 0.990 & 44.20 & 1.93 \\
\cline { 2 - 6 } & Dialectical behavior therapy & 53.73 & 0.799 & 49.53 & 0.990 \\
\cline { 2 - 5 } & Control & 54.80 & 1.26 & 53.93 & 1.44 \\
\hline
\end{tabular}

According to significant f-value calculated for comparison between mean values of two schema therapy and dialectical behavior therapy groups, Lametrix post hoc test was employed for variables, including dimensions of temperament (NS, HA, RD, PS) and character (SD, CO, ST), and cognitive emotion regulation strategies (positive and negative cognitive emotion regulations). Table 4 demonstrates the obtained results.

Table 4. Results of multivariate MANCOVA based on components of temperament, character, and cognitive emotion regulation strategies

\begin{tabular}{|c|c|c|c|c|c|c|}
\hline Diffraction source & $\begin{array}{c}\text { Sum of squares } \\
(\mathrm{SS})\end{array}$ & $\begin{array}{c}\text { Degree of } \\
\text { freedom }(\mathrm{df})\end{array}$ & $\begin{array}{c}\text { Mean square } \\
(\mathrm{MS})\end{array}$ & $\mathrm{F}$ & $\begin{array}{c}\text { Significance level } \\
(\mathrm{P})\end{array}$ & $\begin{array}{c}\text { Mean } \\
\text { differences }\end{array}$ \\
\hline NS & 19.025 & 1 & 19.725 & 21.522 & 0.000 & 2.476 \\
\hline Error & 22.913 & 25 & 0.917 & & & \\
\hline $\mathrm{HA}$ & 15.945 & 1 & 15.945 & 7.848 & 0.000 & 2.226 \\
\hline Error & 50.791 & 25 & 20.032 & & & \\
\hline $\mathrm{RD}$ & 50.904 & 1 & 50.904 & 40.204 & 0.000 & 3.977 \\
\hline Error & 31.654 & 25 & 1.266 & & & \\
\hline PS & 6.490 & 1 & 6.490 & 13.905 & 0.001 & 1.420 \\
\hline Error & 11.668 & 25 & 0.467 & & & \\
\hline SD & 15.930 & 1 & 15.930 & 17.550 & 0.000 & 2.225 \\
\hline Error & 22.692 & 25 & 0.908 & & & \\
\hline $\mathrm{CO}$ & 30.598 & 1 & 30.598 & 18.909 & 0.000 & 3.084 \\
\hline Error & 40.454 & 25 & 1.618 & & & \\
\hline ST & 52.706 & 1 & 52.706 & 49.613 & 0.000 & 4.047 \\
\hline Error & 26.559 & 25 & 1.062 & & & \\
\hline $\begin{array}{l}\text { Positive cognitive emotion } \\
\text { regulation }\end{array}$ & 83.614 & 1 & 83.614 & 21.521 & 0.000 & 5.098 \\
\hline Error & 97.131 & 25 & 3.885 & & & \\
\hline $\begin{array}{l}\text { Negative cognitive } \\
\text { emotion regulation }\end{array}$ & 82.630 & 1 & 82.630 & 51.117 & 0.000 & -5.067 \\
\hline Error & 40.412 & 25 & 1.616 & & & \\
\hline
\end{tabular}

According to Table 4, there was a significant post-test difference between experimental groups that are affected by schema therapy and dialectical behavior therapy regarding values of temperament's components (NS (2.476), HA (2.226), RD (3.977), PS (1.420)), character's components (SD (2.225), 
CO (3.084), ST (4.047)), and positive (5.098) and negative (-5.067) cognitive emotion regulation components. According to the results obtained from data analysis base on the multivariate ANCOVA, it can be concluded (based on the probability of 0.99) the research hypothesis was confirmed; hence, schema therapy and DBT had a different effect on dimensions of temperament and character, as well as cognitive emotion regulation strategies in patients with HIV.

\section{Discussion}

This study was carried out to compare the effect of schema therapy and dialectical behavior therapy on TC's dimensions and cognitive emotion regulation strategies in patients with HIV. Findings were in line with results obtained by Farmani-Shahreza et al., 2017, Belir et al., 2018, Kröger et al., 2013, Khanjari, 2020. Since there is a relationship between inefficient schemas of patients who suffer from HIV with their TC's dimensions and cognitive emotion regulation, any change in their schemas may cause variation in their openness to experience.

In schema therapy, cognitive techniques emphasize the false core beliefs by using various techniques, including pros and cons of coping responses, dialogues between health and schematic aspects, and designing training cards. To this end, the first intervention group received cognitive techniques besides proper relationship therapy and empathic confrontation within four sessions. The second group received experimental techniques of schema therapy, which was performed in the fifth session for the first intervention group. The experimental techniques highly stress the patients' emotions. In this case, the therapist employs limited reparenting to create a safe space playing the role of a good enough parent to review and recalling the bad memories of patients. Finally, at the last step of schema therapy, the pattern of dysfunctional behaviors is broken on contrary to cognitive behavior therapy (CBT) to change those behaviors that are more troublesome. Four types of skills were used in the second intervention group. First, mindfulness skills were taught. Self-awareness helps clients to be aware of their thoughts, impulses, and emotions and to learn how to manage them more effectively. Mindfulness assisted patients to tolerate those thoughts, impulses, and emotions that are intolerable for them. Mindfulness also helped them to perceive those inner experiences that they should not perform but can accept and solve them gradually. Interpersonal efficiency skills were the second category of DBT skills that could mitigate interpersonal distresses that are common among patients who suffer from HIV. Patients were trained to answer the following questions: do you have any specific goal in your interpersonal relationships" do you want to keep or improve self-respect? In this case, they developed interpersonal relationships and mental health. The third category included emotion regulation skills that were used to reduce mood swings. Learning these skills, clients became aware of the relationship between their thoughts, feelings, and behaviors so they could influence one of them by changing another one. The fourth category included distress tolerance skills in which, clients could tolerate crises without involved in problematic behaviors, such as suicide, self-harm, drug abuse, etc. Since schema therapy considers cognitive themes, emotions, and behaviors, this is a more efficient and effective therapy (regarding its multi-dimensional feature) compared to DBT. In this case, schema therapy employs some techniques, including conducting 
a dialogue between current problems and schemas, evaluating the pros and cons of coping responses, challenging schemas, imaginary negotiation, mental imagery, and overcoming barriers to behavioral change, and creating outstanding changes in life.

As we know, schema therapy has emerged to deal with personality and emotional problems by focusing on the history and background of patients. On the other hand, most patients with HIV suffer from a past trauma that has led to unpleasant changes in them. Accordingly, schema therapy was more effective compared to DBT since it focused on the past trauma of such patients that led to their improper choices, such as wrong sexual partners and inefficient coping styles (i.e. drug abuse) used to escape from memories, mental images, and annoying situations. Regarding the high-risk choices of patients with HIV, reward-dependence is one of the temperament components among these patients, which directs them towards novel seeking and lack of harm-avoidance. These patients suffer from much harm due to their weak self-directedness, cooperativeness, and self-transcendence. Schema therapy could identify schemas and linked them to transformational roots by using various techniques within different dimensions. Hence, it not only had a higher effect on components of temperament and character but also made patients use more adaptive methods when facing emotional situations.

There were some constraints in this research. For instance, it was just conducted in one of the clinics in Hamedan, Iran; hence, caution should be taken in generalizing results. Moreover, simple random sampling and time limitation in the data collecting process led to lower accuracy of sampling, which should be taken into account. Therefore, it is recommended to examine the effectiveness of schema therapy and DBT in larger groups of patients with HIV within longer periods. Also, it is suggested to survey the effectiveness of the aforementioned therapies in other emotional and personality components separately and simultaneously.

\section{Conclusion}

It can be stated that schema therapy was a more comprehensive technique rather than DBT. The reason is that schema therapy identifies fundamental patterns of thinking and challenges them, emphasizes the relationship therapy, pays attention to past and present moments, uses pattern-breaking of inefficient behaviors, and employs experimental techniques. Accordingly, schema therapy could take a considerable part in changing temperament, character, and cognitive emotion regulation strategies among patients.

Conflict of interest: The authors state no conflict of interest in the study.

Financial sponsor: The authors acknowledge that they have not received any financial support for all stages of the study, writing and publication of the paper.

Acknowledgment: This paper was adopted from Psychology Dissertation designed by Vahid Hemmati Sabet in Islamic Azad University Kermanshah Branch, which was funded by the authors. We appreciate Islamic Azad University Kermanshah Branch, Hamadan University of Medical Sciences, and all participants of this research. 


\section{References}

Ashraf, M., \& Sitwat, A. (2016). Personality dimensions, positive emotions and coping strategies in the caregivers of people living with HIV in Lahore, Pakistan. International Journal of Nursing Practice, 22(4), 364-374.

Baradaran, M. (2017). Comparison of Temperament and Character Dimensions and Cognitive Emotion Regulation in Anxious and Normal students. Journal of Social Cognition. 6 (1): 67-76. (Persian).

Basharpour, S., Etarod, N., Eini, S. (2017). Temperament and Character Dimensions and depression: the mediating role of cognitive emotion regulation. Thoughts and Behavior in Clinical Psychology, 12(43), 27-36. (Persian).

Belir, S., Ansari Shahidi, M., Mohammadi, S. (2018). Efficacy of Dialectical Behavior Therapy on Risky Behaviors, Depression, Anxiety and Stress in Patients with AIDS. hrjbaq. 3 (3) :155-162. (Persian).

Brandt, C., Zvolensky, M. J., Woods, S. P., Gonzalez, A., Safren, S. A., \& O’Cleirigh, C. M. (2017). Anxiety symptoms and disorders among adults living with HIV and AIDS: A critical review and integrative synthesis of the empirical literature. Clinical psychology review, 51, 164-184.

Cane, T. P. C. (2018). Facilitating and supporting HIV+ parenthood: Lessons for developing the advocate role of voluntary HIV support services workers. Sexual \& reproductive healthcare, 16, 186191.

Dadomo, H., Panzeri, M., Caponcello, D., Carmelita, A., \& Grecucci, A. (2018). Schema therapy for emotional dysregulation in personality disorders: A review. Current opinion in psychiatry, 31(1), 4349.

Farmani-Shahreza, S., Ghaedniay-jahromi, A., Mohammad-Taghi Nasab M., Niknezhad, M., Darharaj, M., Sadeghi, M., et al. (2017). Comparison of Cognitive Emotion Regulation Strategies and Emotional Schemas in People with Human Immunodeficiency Virus and Normal People. IJPN. 4 (6) :30-40. (Persian).

Gross, J. J. (2015). The extended process model of emotion regulation: Elaborations, applications, and future directions. Psychological Inquiry, 26(1), 130-137.

Hajializadeh, K., Norizadeh, B. (2018). The Effectiveness Of Dialectical Behavioral Therapy On Reducing Anxiety And Improving Social Functioning Of Patients With Diabetes. ijdld. 17 (3) :130138. (Persian).

Jalali, F., Hasani, A., Hashemi, S. F., Kimiaei, S. A., \& Babaei, A. (2019). Cognitive group therapy based on schema-focused approach for reducing depression in prisoners living with HIV. International journal of offender therapy and comparative criminology, 63(2), 276-288.

Khanjari, C., Khajevand, A. (2020). The Effectiveness Schema Therapy (ST) on Temperament and Character dimensions, Food Craving among Obese Women with Type 2 Diabetes. Journal of Advances in Cognitive Sciences. 22 (2). (Persian).

Khodabandelow, S., Najafi, M., Rahimian Boogar, I. (2018). The Effectiveness of Schema Mode Therapy on the Self-Esteem in Narcissistic Personality Disorder: A Single-Subject Research. Clinical Psychology Studies, 8(30), 65-101. (Persian).

King, B., \& Winchester, M. S. (2018). HIV as social and ecological experience. Social science \& medicine, 208, 64-71. 
Kröger, C., Harbeck, S., Armbrust, M., \& Kliem, S. (2013). Effectiveness, response, and dropout of dialectical behavior therapy for borderline personality disorder in an inpatient setting. Behaviour Research and Therapy, 51(8), 411-416.

M Svrakic, D., \& Robert Cloninger, C. (2012). Pharmacotherapy and the psychobiological model of personality: implications for DSM-5. Current Psychopharmacology, 1(2), 122-136.

Rajabi, S., Nazarpour, M., Tabnak, F. (2017). Predict Cognitive Emotion Regulation Strategies Using the Flexibility of Mind, Retention and Continuous Performance in Adolescents. Journal of Neuropsychology. 3 (9): 9-24. (Persian).

Sahraian, K., Peyvastegar, M., Khosravi, Z., Habibi, M. (2019). Comparing cognitive rehabilitation and mixed treatment of cognitive rehabilitation with emotion regulation on quality of life in HIV patients with neurocognitive disorders. QUARTERLY JOURNAL OF HEALTH PSYCHOLOGY, 8(29), 2551. (Persian).

SayyahBargard, M., Olapour, A., Ardame, A., Shahidi, S., Yaghoobi Askarabad, E. (2014). Prediction of Mental Health and Academic Performance of students within Cognitive Emotional Regulation Strategies in Medical Sciences University of Jundishapur in Ahvaz. Educational Development of Judishapur. 5(1), 37-44. (Persian).

Schadé, A., van Grootheest, G., \& Smit, J. H. (2013). HIV-infected mental health patients: characteristics and comparison with HIV-infected patients from the general population and noninfected mental health patients. Bmc Psychiatry, 13(1), 35.

Teimory, S., Ghafariyan, G., Yazdanpanah, F. (2019). The Effectiveness of Dialectical Behavioral Therapy on Marital Satisfaction in Couples Conflict. Research in Clinical Psychology and Counseling. 8(2), 101-115. (Persian).

Young, J. E., Klosko, J. S., \& Weishaar, M. E. (2003). Schema therapy: A practitioner's guide Guilford Press. New York, NY. 